\title{
Poisson-Vlasov: stochastic representation and numerical codes
}

\author{
E. Floriani ${ }^{1}$, R. Lima ${ }^{1}$, and R. Vilela Mendes ${ }^{2,3, a}$
}

1 Centre de Physique Théorique, CNRS Luminy, case 907, 13288 Marseille Cedex 9, France

2 Centro de Fusão Nuclear, EURATOM/IST Association, Instituto Superior Técnico, Av. Rovisco Pais 1, 1049-001 Lisboa, Portugal

3 CMAF, Complexo Interdisciplinar, Universidade de Lisboa, Av. Gama Pinto 2, 1649-003 Lisboa, Portugal

Received 27 November 2007

Published online 12 December 2007 - (c) EDP Sciences, Società Italiana di Fisica, Springer-Verlag 2007

In equations (7) and (12)

$$
\frac{\overrightarrow{\xi_{2}} \cdot \hat{\xi_{1}^{\prime}}}{\gamma\left(\left|\xi_{2}\right|\right)}
$$

should be replaced by

$$
\frac{\left(\overrightarrow{\xi_{2}}+s \frac{\overrightarrow{\xi_{1}}}{\gamma\left(\left|\xi_{2}\right|\right)}\right) \cdot \hat{\xi}_{1}^{\prime}}{\gamma\left(\left|\xi_{2}+s \frac{\xi_{1}}{\gamma\left(\left|\xi_{2}\right|\right)}\right|\right)} .
$$

This replacement in no way affects the existence of the stochastic process $I$, nor the representation proposition $\mathbf{1}$ which remains valid. However, although the stochastic process II, defined by equation (20), is still a well-defined and convergent process, it does not provide a complete representation for the solutions of the Poisson-Vlasov equation. Therefore proposition 2 is not correct.

This in no way affects the construction of the probabilities needed for the numerical evaluation of the solutions, derived in Section 3. Also the numerical results for the examples studied in Section 3 are qualitatively the same, because of the small contribution of the trees that are neglected when instead of the process I one considers process II.

\footnotetext{
a e-mail: vilela@cii.fc.ul.pt
} 